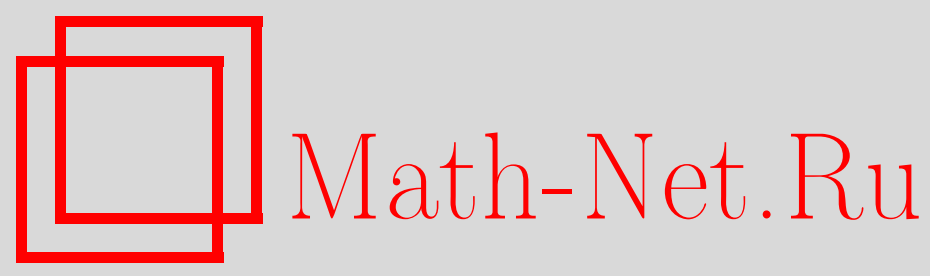

Е. А. Тимофеев, Состоятельная оценка энтропии мер и динамических систем, Матем. заметки, 2005, том 77, выпуск 6, 903-916

DOI: https://doi.org/10.4213/mzm2546

Использование Общероссийского математического портала Math-Net.Ru подразумевает, что вы прочитали и согласны с пользовательским соглашением http://www . mathnet.ru/rus/agreement

Параметры загрузки:

IP : 54.196 .121 .252

26 апреля 2023 г., 03:46:40

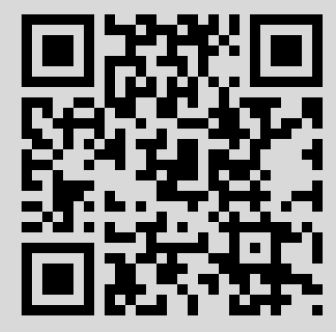




\title{
СОСТОЯТЕЛЬНАЯ ОЦЕНКА ЭНТРОПИИ МЕР И ДИНАМИЧЕСКИХ СИСТЕМ
}

\author{
Е. А. Тимофеев
}

\begin{abstract}
В работе описан новый инвариант мер и динамических систем, названный $\mathrm{cmamə \vartheta -}$ mponuей. Для статэнтропии построена статистическая оценка, которая вычисляется без построения вспомогательных оценок меры. Доказано, что предлагаемая статистическая оценка является состоятельной при достаточно общих ограничениях. Показано, что для точно размерностных мер статэнтропия совпадает с хаусдорфовой размерностью меры, а для эргодических динамических систем статэнтропия совпадает с метрической энтропией отображения.
\end{abstract}

Библиографоия: 12 названий.

\section{1. Введение}

Нахождение (метрической) энтропии мер и динамических систем представляет большой как прикладной, так и теоретический интерес (см., например, [1]-[4]). При вычислении оценок энтропии по заданньм траекториям динамической системы основной трудностью является то, что частоты попадания точек траектории в различные области очень резко отличаются. Однако большинство ранее известных методов вычисления энтропии основано на вспомогательньх оценках инвариантной меры (вероятностей попадания в небольшие области), которые являются заведомо не точными для некоторых областей. Предлагаемые в работе оценки энтропии не требуют вспомогательньх оценок меры.

Рассматриваемая задача состоит в следующем. Пусть $\Omega$ - компактное метрическое пространство с метрикой $\rho$ и с борелевской вероятностной мерой $\mu$. Пусть даны $n$ независимых случайных величин $\xi_{1}, \xi_{2}, \ldots, \xi_{n}$, принимающих значения в $\Omega$ и имеющих общее распределение - меру $\mu$. В работе предлагается семейство состоятельных статистических оценок нового инварианта мер, названного статэнтропией.

Показано, что для точно размерностных мер статэнтропия совпадает с хаусдорфовой размерностью меры.

В настоящей статье основной областью применения оценок является задача нахождения энтропии динамической системы. В этом случае $\Omega$ является пространством последовательностей, а статэнтропия пропорциональна энтропии сдвига, если сдвиг эргодичен (коэффициент пропорциональности зависит от метрики). Подчеркнем, что в прикладных задачах инвариантная мера обычно не известна, а известными являются 
только траектории, поэтому в таких задачах рассматриваемая постановка представляется довольно естественной.

Суть предлагаемого подхода состоит в том, что статэнтропия является обратной величиной коэффициента (с отрицательным знаком) при асимптотике логарифма среднего минимального расстояния между траекториями, когда число траекторий стремится $\mathrm{K}$ бесконечности.

Для иллюстрации нашего подхода приведем пример. Пусть $\Omega-s$-мерньй куб с обычной метрикой и лебеговой мерой $\mu$. Пусть $\xi_{1}, \xi_{2}, \ldots, \xi_{n}$ - независимые точки равномерно распределенные в $\Omega$, тогда статэнтропия совпадает с размерностью $s$, а среднее минимальное расстояние $r_{n}$ между $n$ точками $\xi_{1}, \xi_{2}, \ldots, \xi_{n}$ в $s$-мерном кубе равняется $\mathscr{O}\left(n^{-1 / s}\right)$. Статистическая оценка статэнтропии равна $-\ln n / \ln r_{n}$ и сходится к $s$.

Совпадение статэнтропии и энтропии представляется естественньм, если представить энтропию как “коэффициент при асимптотике логарифма числа различных типов траекторий” [3, гл. 14] и применить свойство равномерной распределенности траекторий (теорема Шеннона-МакМиллана-Бреймана для эргодических отображений).

Построение статистических оценок энтропии без вспомогательных оценок меры было начато в работе Добрушина [5] (см. также [6]). Настоящая статья продолжает подход, начатый в работах [7], [8].

\section{2. Определения, ограничения и обозначения}

2.1. Определение статэнтропии меры. Пусть дано компактное метрическое пространство $\Omega$ с метрикой $\rho=\rho(x, y)$ и с борелевской вероятностной мерой $\mu$. Для простоты будем считать, что $\operatorname{diam} \Omega \leqslant 1$.

Прежде чем приводить определение, введем вспомогательные функцию и функционал.

Через $r=\nu(t, x)$ обозначим обобщенную обратную функцию к функции $t=\mu(B(x, r))$ (при заданном $x$ ). Здесь и далее через $B(x, r)$ будем обозначать открытьй шар радиуса $r$ с центром в точке $x$. Функция $r=\nu(t, x)$ задает радиус шара меры $t$ с центром в точке $x$ и при заданном $x$ определяется следуюшим образом:

$$
\nu(t, x)=\inf \{r: \mu(B(x, r)) \geqslant t\}
$$

Подчеркнем, что функции $t=\mu(B(x, r))$ и $r=\nu(t, x)$ могут иметь участки постоянства и точки разрыва (постоянство одной функции соответствует разрыву другой).

Через $N(u)$ обозначим функционал от функции $u(x)$, равньй

$$
N(u)=-\int_{\Omega} \ln (|u(x)|) d \mu(x) .
$$

Нижней и верхней статэнтропиями меры $\mu$ относительно метрики $\rho$ будем назьвать величины

$$
\underline{\eta}(\mu, \rho)=\varliminf_{t \rightarrow 0+} \frac{-\ln t}{N(\nu(t, \cdot))}, \quad \bar{\eta}(\mu, \rho)=\varlimsup_{t \rightarrow 0+} \frac{-\ln t}{N(\nu(t, \cdot))} .
$$

Если $\underline{\eta}(\mu, \rho)=\bar{\eta}(\mu, \rho)=\eta(\mu, \rho)$, то величину $\eta(\mu, \rho)$ будем назьвать статэнтропией меры $\mu$. 
2.2. Статистическая оценка статэнтропии меры. Пусть $\xi_{1}, \xi_{2}, \ldots, \xi_{n}$ - независимые одинаково распределенные случайные величины, принимающие значения в $\Omega$ и имеющие общее распределение - меру $\mu$.

Статистическая оценка $\eta_{n}(\rho)$ статэнтропии строится следующими простыми вычислениями:

1) находим вспомогательную случайную величину

$$
r_{n}^{(k)}=-\frac{1}{n} \sum_{j=1}^{n} \ln \left(\min _{i: i \neq j}^{(k)} \rho\left(\xi_{i}, \xi_{j}\right)\right)
$$

где $\min ^{(k)}\left\{x_{1}, \ldots, x_{N}\right\}=x_{k}$, если $x_{1} \leqslant x_{2} \leqslant \cdots \leqslant x_{N} ;$

2) полагаем оценкой статэнтропии случайную величину

$$
\eta_{n}^{(k)}(\rho)=\frac{\ln n}{r_{n}^{(k)}}
$$

Отметим, что $\eta_{n}^{(k)}(\rho) \leqslant \eta_{n}^{(k+1)}$, поэтому значения $k>1$ нужны только для дополнительной проверки. Далее будет показано, что в пределе при $n \rightarrow \infty$ оценка не зависит от $k$.

Подчеркнем, что мера $\mu$ не используется при построении оценки - она участвует только как распределение случайных величин $\xi_{1}, \xi_{2}, \ldots, \xi_{n}$.

2.3. Вспомогательные обозначения и ограничения. Математическое ожидание и дисперсию случайной величины $\xi$ будем обозначать через $\mathrm{E} \xi$ и $\mathrm{D} \xi$ соответственно.

Напомним, что нижней и верхней точечными (pointwise) размерностями меры $\mu$ в точке $x$ назьваются величины

$$
\underline{d}_{\mu}(x)=\varliminf_{u \rightarrow 0} \frac{\ln \mu(B(x, u))}{\ln u}, \quad \bar{d}_{\mu}(x)=\varlimsup_{u \rightarrow 0} \frac{\ln \mu(B(x, u))}{\ln u} .
$$

Если $\underline{d}_{\mu}(x)=\bar{d}_{\mu}(x)=d_{\mu}(x)$, то величина $d_{\mu}(x)=d(x)$ назьвается точечной размерностью меры $\mu$ в точке $x$.

Будем требовать, чтобы мера $\mu$ и метрика $\rho$ удовлетворяли условию, которое состоит в том, что

$$
\exists \bar{d} \geqslant \underline{d}>0: \underline{d} \leqslant \underline{d}_{\mu}(x) \leqslant \bar{d}_{\mu}(x) \leqslant \bar{d} \quad \text { для } \mu \text {-почти всех } \quad x \in \Omega .
$$

Меру $\mu$ будем называть точно размерностной (exact dimensional) (см., например, $[4$, c. 44]), если для $\mu$-почти всех точек $x \in \Omega$ существует и постоянна $d(x)=d$. Для точно размерностных мер число $d$ совпадает с размерностью Хаусдорфа меры $\mu$ (см., например, [4, с. 42]), которую будем обозначать через $\operatorname{dim}_{H} \mu$. 
2.4. Пространство последовательностей. Одним из основных примеров рассматриваемых пространств $\Omega$ являются компакты в пространстве последовательностей $S^{\mathbb{N}}$, где множество $S=\{0,1,2, \ldots, s-1\}, \mathbb{N}=\{0,1,2, \ldots, n, \ldots\}$. Точки пространства $S^{\mathbb{N}}$ будем обозначать через

$$
x=\left(x_{0}, x_{1}, \ldots, x_{n}, \ldots\right), \quad x_{i} \in S
$$

Снабдим $S^{\mathbb{N}}$ метрикой

$$
\rho(x, y)=\theta^{-n}, \quad \text { где } n: x_{n} \neq y_{n}, \quad x_{0}=y_{0}, \ldots, x_{n-1}=y_{n-1},
$$

$\theta>1$ - некоторьй параметр. Отметим, что выбор метрики $\rho$ обусловлен следующими ее свойствами:

1) в этой метрике шары являются цилиндрами;

2 ) для вычисления $\rho(x, y)$ достаточно знания только конечного числа элементов последовательностей $x$ и $y$.

Первое свойство существенно облегчает доказательства и вычисления. Второе свойство позволяет точно находить $\rho(x, y)$ в прикладных задачах, в которых траектории задаются конечным числом точек.

Цилиндры в пространстве последовательностей $S^{\mathbb{N}}$ будем обозначать через

$$
C_{n}(x)=\left\{y \in S^{\mathbb{N}}: y_{i}=x_{i}, i=0,1, \ldots, n-1\right\}
$$

2.5. Символические динамические системы. Пусть $X$ - компактное метрическое пространство с метрикой $d, T: X \rightarrow X$ - непрерьвное отображение. Будем считать, что отображение $T$ имеет инвариантную меру $P$, которая является борелевской и вероятностной.

Пусть $\alpha$ - конечная случайная величина на вероятностном пространстве $(X, P)$, которая принимает значения из множества $S=\{0,1,2, \ldots, s-1\}$. Случайная величина $\alpha$ задает разбиение $\mathscr{A}$ пространства $X$ на множества $A_{i}=\{x \in X: \alpha(x)=i\}, i \in S$.

Рассмотрим пространство последовательностей $S^{\mathbb{N}}$ с метрикой $\rho$, заданной в $(8)$, с непрерьвньп преобразованием - сдвигом $\sigma$, где

$$
\sigma\left(x_{0}, x_{1}, \ldots, x_{n}, \ldots\right)=\left(x_{1}, x_{2}, \ldots, x_{n+1}, \ldots\right)
$$

Определим символическую динамическую систему $\left(\Omega, \sigma, \mu_{\alpha}\right)$, построенную по динамической системе $(X, T, P)$ и по случайной величине $\alpha$, положив

$$
\Omega=\left\{\omega: \omega=\left(\alpha(x), \alpha(T(x)), \ldots, \alpha\left(T^{n}(x)\right), \ldots\right) \quad \forall x \in X\right\}
$$

Ясно, что $\sigma(\Omega) \subset \Omega$.

Инвариантная мера $P$ преобразования $T$ порождает инвариантную меру $\mu$ сдвига $\sigma$ на $\Omega$ стандартным образом. По построению мера $\mu$ является вероятностной, а поскольку для рассматриваемой метрики $\rho$ цилиндры являются шарами, то $\mu$-борелевская мера. 
2.6. Определение статэнтропии динамической системы. Пусть для динамической системы $(X, T, P)$ выбрана конечная случайная величина $\alpha$ (разбиение) и построена символическая динамическая система $\left(\Omega, \sigma, \mu_{\alpha}\right)$ с метрикой $\rho$, заданной в $(8)$.

Нижней и верхней статэнтропиями динамической системы $(X, T, P)$ будем назьвать величины

$$
\underline{S H}(T, P)=\sup _{\alpha} \underline{\eta}\left(\mu_{\alpha}, \rho\right), \quad \overline{S H}(T, P)=\sup _{\alpha} \bar{\eta}\left(\mu_{\alpha}, \rho\right),
$$

где верхняя грань берется по всем конечным случайным величинам $\alpha$.

Если $\underline{S H}(T, P)=\overline{S H}(T, P)=S H(T, P)$, то величину $S H(T, P)$ будем называть статэнтропией динамической системы.

Подчеркнем, что это определение аналогично определению метрической энтропии $h_{P}(T)$ динамической системы $(X, T, P)$ по энтропии $h(T, \mathscr{A})$ на один знак разбиения $\mathscr{A}$, соответствующего случайной величине $\alpha$ (см., например, [3, лекция 14]).

Кроме трудностей таких же, как и у энтропии: конечность супремума по $\alpha$ и его достижимость на конечном разбиении, здесь есть и дополнительная, связанная с отсутствием предела по разбиению. Однако для эргодических динамических систем эта трудность преодолима.

\section{3. Свойства статэнтропии меры}

В этом разделе будет доказана инвариантность статэнтропии $\eta(\mu, \rho)$ при билипшицевой замене мер и метрик, а также ряд других ее свойств.

УТВЕРЖДЕНИЕ 1. Справедливы неравенства

$$
\left(\int_{\Omega} \frac{d \mu(x)}{\underline{d}(x)}\right)^{-1} \leqslant \underline{\eta}(\mu, \rho) \leqslant \bar{\eta}(\mu, \rho) \leqslant\left(\int_{\Omega} \frac{d \mu(x)}{\bar{d}(x)}\right)^{-1} .
$$

ДоКАЗАТЕЛЬСТВо. Из определения (3) следует, что

$$
\underline{\eta}(\mu, \rho)^{-1}=\varlimsup_{t \rightarrow 0+} \frac{N(\nu(t, \cdot))}{-\ln t} .
$$

Применив лемму Фату [9, п. 2.4.9], получим

$$
\underline{\eta}(\mu, \rho)^{-1} \leqslant \int_{\Omega} \varlimsup_{t \rightarrow 0+} \frac{\ln (\nu(t, x))}{\ln t} d \mu(x) .
$$

Из определения локальных размерностей (6) следует, что

$$
\varlimsup_{t \rightarrow 0+} \frac{\ln (\nu(t, x))}{\ln t}=\left(\underset{t \rightarrow 0+}{\lim } \frac{\ln t}{\ln (\nu(t, x))}\right)^{-1}=\left({\underset{\lim }{r \rightarrow 0+}}_{t} \frac{\ln \mu(B(x, r))}{\ln r}\right)^{-1}=\underline{d}(x)^{-1} .
$$

Подставляя в (12), получим первое неравенство в (11).

Аналогично доказывается и оценка сверху. 
СлЕДСТВИЕ 1. Пусть мера $\mu$ является точно размерностной, тогда существует статэнтропия $\eta(\mu, \rho)$ u $\eta(\mu, \rho)=\operatorname{dim}_{H} \mu$.

УТВЕРЖДЕНИЕ 2. Пусть $\Omega_{1}, \Omega_{2}$ - компактные метрические пространства $c$ метриками $\rho_{1}$ и $\rho_{2}$, пусть $\mu_{1}$ - борелевская вероятностная мера на $\Omega_{1}$, пусть $F: \Omega_{1} \rightarrow \Omega_{2}$ - билипиицевый гомеоморфизм, и пусть существует статәнтропия $\eta\left(\mu_{1}, \rho_{1}\right)$ для тройки $\left(\Omega_{1}, \rho_{1}, \mu_{1}\right)$. Тогда статэнтропия существует для тройки $\left(\Omega_{2}, \rho_{2}, \mu_{2}=\mu_{1} \circ F^{-1}\right)$ и равна $\eta\left(\mu_{1}, \rho_{1}\right)$.

ДокАЗАТЕЛЬСТво. В пространстве $\Omega_{2}$ имеем

$$
\mu_{2}(B(F(x), u))=\mu_{1}\left(\left\{y \in \Omega_{1}: \rho_{2}(F(y), F(x)) \leqslant u\right\}\right) \quad \forall u \geqslant 0, \quad x \in \Omega_{1}
$$

Поскольку $F(x)$ - билипшицевьй гомеоморфизм компактных пространств, то для некоторой константы $c>1$ имеем

$$
c^{-1} \rho_{1}(x, y) \leqslant \rho_{2}(F(x), F(y)) \leqslant c \rho_{1}(x, y) \quad \forall x, y \in \Omega_{1} \text {. }
$$

Поэтому

$$
\mu_{1}\left(B\left(x, c^{-1} u\right)\right) \leqslant \mu_{2}(B(F(x), u)) \leqslant \mu_{1}(B(x, c u)) .
$$

Для обратных функций имеем

$$
c^{-1} \nu_{1}(t, x) \leqslant \nu_{2}(t, F(x)) \leqslant c \nu_{1}(t, x) .
$$

Подставляя эти оценки в (2), получим

$$
N\left(\nu_{1}(t, \cdot)\right)-\ln c \leqslant N\left(\nu_{2}(t, \cdot)\right) \leqslant N\left(\nu_{1}(t, \cdot)\right)+\ln c .
$$

Следовательно, статэнтропия существует для тройки $\left(\Omega_{2}, \rho_{2}, \mu_{2}=\mu_{1} \circ F^{-1}\right)$ и равна той же самой функции $\eta\left(\mu_{1}, \rho_{1}\right)$.

Доказанное утверждение не только переносит статэнтропию на другие пространства, но и показьвает ее инвариантность при гладкой замене метрики.

СлЕДСТВИЕ 2. Пусть $\Omega$ - компактное метрическое пространство, пусть $\mu$ борелевская вероятностная мера на $\Omega$, пусть $\rho_{1}$ и $\rho_{2}$ - две әквивалентные метрики, т.е. для некоторой константы $c>1$

$$
c^{-1} \rho_{1}(x, y) \leqslant \rho_{2}(x, y) \leqslant c \rho_{1}(x, y) \quad \forall x, y \in \Omega
$$

и пусть существует статэнтропия $\eta\left(\mu, \rho_{1}\right)$. Тогда статэнтропия существует для тройки $\left(\Omega, \rho_{2}, \mu\right)$ и $\eta\left(\mu, \rho_{2}\right)=\eta\left(\mu, \rho_{1}\right)$. 


\section{4. Свойства статистической оценки}

Пусть $\xi_{1}, \xi_{2}, \ldots, \xi_{n}$ - независимые одинаково распределенные случайные величины, принимающие значения в $\Omega$ и имеющие общее распределение - меру $\mu$.

В этом разделе будет доказано, что математическое ожидание статистической оценки $\eta_{n}^{(k)}(\rho)$ стремится к статэнтропии $\eta(\mu, \rho)$ при $n \rightarrow \infty$, и будет доказана состоятельность оценки $\eta_{n}^{(k)}(\rho)$.

Условия, накладываемые на меру $\mu$ и метрику $\rho$ в этом разделе, - это условие (7) и условие существования статэнтропии $\eta(\mu, \rho)$ меры $\mu$ относительно метрики $\rho$.

4.1. Распределение расстояния до $k$-й ближайшей точки. Обозначим через $R_{n, i}^{(k)}$ случайную величину, определяемую по формуле

$$
R_{n, i}^{(k)}=\min _{j: j \neq i}{ }^{(k)} \rho\left(\xi_{j}, \xi_{i}\right), \quad i=1,2, \ldots, n .
$$

Приведем необходимые для дальнейшего результаты о распределении величин $R_{n, i}^{(k)}$, которые доказаны в работе [8]. Отметим, что функция $\mu(B(x, r))$ (которая обозначается в [8] через $F(r, x))$ задает распределение случайной величины $\rho(\xi, x)$.

Введем две вспомогательные условные вероятности:

$$
Q_{n, k}(u, y)=\mathrm{P}\left\{R_{n, i}^{(k)}=u \mid \xi_{i}=y, \rho\left(y, \xi_{i^{\prime}}\right)=u\right\}
$$

- вероятность того, что точки $\xi_{m}$ расположены так, что в шар $B(y, u)$ попало ровно $k-1$ точек, при условии того, что $\xi_{i}=y$ и на гранище шара $B(y, u)$ лежит точка $\xi_{i^{\prime}}$;

$$
Q_{n, k}^{(2)}(u, v, y, z)=\mathrm{P}\left\{R_{n, i}^{(k)}=u, R_{n, j}^{(k)}=v \mid \xi_{i}=y, \xi_{j}=z, \rho\left(y, \xi_{i^{\prime}}\right)=u, \rho\left(z, \xi_{j^{\prime}}\right)=v\right\}
$$

- вероятность того, что точки $\xi_{m}$ расположены так, что в каждый из шаров $B(y, u)$, $B(z, v)$ попало ровно по $k-1$ точке, при условии того, что $\xi_{i}=y, \xi_{j}=z$, на границе $B(y, u)$ лежит точка $\xi_{i^{\prime}}$ и на гранище $B(z, v)$ лежит точка $\xi_{j^{\prime}}$.

Вероятность $Q_{n, k}(u, y)$ легко вычисляется:

$$
Q_{n, k}(u, x)=\left(\begin{array}{l}
n-2 \\
k-1
\end{array}\right) \mu(B(x, u))^{k-1}(1-\mu(B(x, u)))^{n-k-1} .
$$

Для вероятности $Q_{n, k}^{(2)}(u, v, y, z)$ в [8] получены следующие оценки в зависимости от взаимного расположения точек $y, z$ и точек, лежаших на границах шаров.

ЛЕмма 1. Пусть неравенства условия (7) выполнены в точках $y, z \in \Omega$, пусть а такое, что $0<a<\min \{\underline{d}, 1 / \bar{d}\}$. Тогда при $n \rightarrow \infty$ имеем

$$
Q_{n, k}^{(2)}(u, v, y, z) \leqslant\left(1+\mathscr{O}\left(n^{-a \underline{d}}\right)\right) Q_{n, k}(u, y) Q_{n, k}(v, z),
$$

если $\rho(y, z)>2 n^{-a}, \quad u, v<n^{-a}$;

$$
Q_{n, k}^{(2)}(u, v, y, z)=\mathscr{O}\left(c^{n^{b}}\right), \quad b>0, \quad 0<c<1
$$

если $u>n^{-a}$ илu $v>n^{-a}$;

$$
Q_{n, k}^{(2)}(u, v, y, z)=\mathscr{O}\left(\max _{k / 2-1 \leqslant k_{1}, k_{2} \leqslant k} Q_{n / 2-k, k_{1}}(u, y) Q_{n / 2-k, k_{2}}(v, z)\right),
$$

при любьх $y, z, u, v$. 
4.2. Несмещенность оценки $\eta_{n}^{(k)}(\rho)$. Покажем, что оценка $\eta_{n}^{(k)}(\rho)$ является асимптотически несмещенной.

Лемма 2. Пусть неравенства условия (7) выполнены в точке $x \in \Omega$. Тогда существует

$$
\mathrm{E}\left[-\ln \left(R_{n, i}^{(k)}\right) \mid \xi_{i}=x\right]=(n-1)\left(\begin{array}{l}
n-2 \\
k-1
\end{array}\right) \int_{0}^{1}-\ln (\nu(t, x)) t^{k-1}(1-t)^{n-k-1} d t
$$

ДокАЗАТЕЛЬСТво. Из определения условной плотности $Q_{n, k}(u, x)$ имеем

$$
\mathrm{E}\left[-\ln \left(R_{n, i}^{(k)}\right) \mid \xi_{i}=x\right]=\int_{0}^{\infty}-\ln (u) Q_{n, k}(u, x) d_{u} \mu(B(u, x))
$$

Этот интеграл заменой $t=\mu(B(u, x))$ (см., например, $[9$, п. 2.5.18, (3)]) сводится к интегралу

$$
\int_{0}^{\infty}-\ln (\nu(t, x)) Q_{n, k}(\nu(t, x), x) d t
$$

который совпадает с формулой (18) после подстановки $Q_{n, k}(u, y)$ из (14).

Интеграл в (18) имеет особенность только в 0. Действительно, из неравенств (7) следует, что

$$
\mu(B(u, x)) \geqslant c_{1} u^{\underline{d}}, \quad c_{1}>0
$$

поэтому

$$
-\ln \nu(t, x)=\mathscr{O}(-\ln t)
$$

Следовательно, интеграл в (18) существует при любом $k$.

СЛЕДСТВИЕ 3. Пусть выполнено условие (7). Тогда

$$
\mathrm{E} r_{n}^{(k)}=\mathrm{E}\left(-\ln R_{n, i}^{(k)}\right)=(n-1)\left(\begin{array}{l}
n-2 \\
k-1
\end{array}\right) \int_{0}^{1} N(\nu(t, \cdot)) t^{k-1}(1-t)^{n-k-1} d t
$$

ДокАЗАТЕЛьСтво. Проинтегрировав равенство (18) по мере $\mu$ и подставив (2), получим второе равенство в (19). В силу обозначений (13) имеем

$$
r_{n}^{(k)}=\frac{1}{n} \sum_{i=1}^{n}-\ln \left(R_{n, i}^{(k)}\right)
$$

Отсюда следует первое равенство в (19).

ТЕОРема 1. Пусть выполнено условие (7), пусть существует $\eta(\mu, \rho)$. Тогда сущ, ествует

$$
\lim _{n \rightarrow \infty} \frac{\mathrm{E} r_{n}^{(k)}}{\ln n}=\eta(\mu, \rho)^{-1} .
$$


ДоКАЗАТЕЛЬСТво. Из условия существования статэнтропии $\eta=\eta(\mu, \rho)$ следует, что для любого $\varepsilon>0$ для достаточно малых $t$ справедливы неравенства

$$
\frac{-\ln t}{\eta+\varepsilon} \leqslant N(\nu(t, \cdot)) \leqslant \frac{-\ln t}{\eta-\varepsilon} .
$$

Подставляя эти оценки в $\mathrm{E} r_{n}^{(k)}$, задаваемое формулой (19), и применяя формулу [10, п. 4.253.1], получим, что

$$
\frac{1}{\eta+\varepsilon} \sum_{i=k}^{n-1} \frac{1}{i} \leqslant \mathrm{E} r_{n}^{(k)} \leqslant \frac{1}{\eta-\varepsilon} \sum_{i=k}^{n-1} \frac{1}{i} .
$$

Следовательно, для любого $\varepsilon>0$ при достаточно большом $n$ справедливы неравенства

$$
\frac{1}{\eta+\varepsilon} \ln n \leqslant \mathrm{E} r_{n}^{(k)} \leqslant \frac{1}{\eta-\varepsilon} \ln n
$$

Доказанное утверждение, в частности, показывает, что оценка $\eta_{n}^{(k)}(\rho)$ не зависит от параметра $k$ при достаточно большом $n$.

СлЕДСтвиЕ 4. Пусть выполнено условие (7). Тогда

$$
\mathrm{E} \ln ^{2} R_{n, i}^{(k)}=\mathscr{O}\left(\ln ^{2} n\right)
$$

ДОКАЗАТЕЛЬСТво. Из условия (7) следует, что

$$
\ln \nu(t, x)=\mathscr{O}(-\ln t)
$$

Подставляя эти оценки в

$$
\mathrm{E} \ln ^{2} R_{n, i}^{(k)}=(n-1)\left(\begin{array}{l}
n-2 \\
k-1
\end{array}\right) \int_{0}^{1} \int_{\Omega} \ln ^{2} \nu(t, x) d \mu(x) t^{k-1}(1-t)^{n-k-1} d t
$$

и применяя формулу [10, п. 4.261.21], получим (21).

4.3. Состоятельность оценки $\eta_{n}^{(k)}(\rho)$.

Теорема 2. Пусть существует $\eta=\eta(\mu, \rho)$ и выполнено условие (7). Тогда для $c<\min \left\{\underline{d}^{2}, \underline{d} / \bar{d}, 1\right\}$

$$
\mathrm{D}\left(\frac{r_{n}^{(k)}}{\ln n}\right)=\mathscr{O}\left(n^{-c}\right)
$$

ДоКАЗАТЕЛЬСТво. Оценим ковариацию и дисперсию случайных величин $-\ln \left(R_{n, i}^{(k)}\right)$. Существование $\mathrm{E}\left(-\ln R_{n, i}^{(k)}\right)$ следует из леммы $2 . \quad$ Существование $\mathrm{E}\left(\ln ^{2} R_{n, i}^{(k)}\right)$ доказано в следствии 4 . 
Лемма 3. Пусть существует $\eta=\eta(\mu, \rho)$ и выполнено условие (7). Тогда для любого $\delta<\underline{d} \min \{\underline{d}, 1 / \bar{d}\}$

$$
\operatorname{Cov}\left(-\ln R_{n, i}^{(k)},-\ln R_{n, j}^{(k)}\right)=\mathscr{O}\left(n^{-\delta}\right) \ln ^{2} n, \quad i \neq j .
$$

ДокАЗАТЕльство. Выберем параметр $a<\min \{\underline{d}, 1 / \bar{d}\}$.

Пусть $S$ - событие, состоящее в том, что $\rho\left(\xi_{j}, \xi_{i}\right)<2 n^{-a}$, тогда

$$
\mathrm{P}\{S\}=\int_{\Omega} \mu\left(B\left(2 n^{-a}, y\right)\right) d \mu(y) .
$$

Применяя неравенства (7), получим

$$
\mathrm{P}\{S\}=\mathscr{O}\left(n^{-a \underline{d}}\right) .
$$

По формуле полной вероятности имеем

$$
\mathrm{E}\left(\ln R_{n, i}^{(k)} \ln R_{n, j}^{(k)}\right)=\mathrm{E}\left(\ln R_{n, i}^{(k)} \ln R_{n, j}^{(k)} \mid S\right) \mathrm{P}\{S\}+\mathrm{E}\left(\ln R_{n, i}^{(k)} \ln R_{n, j}^{(k)} \mid \bar{S}\right)(1-\mathrm{P}\{S\}) .
$$

Для оценки условного математического ожидания $\mathrm{E}\left(\ln R_{n, i}^{(k)} \ln R_{n, j}^{(k)} \mid S\right)$ применим равенство (17) из леммы 1. Умножив равенство (17) на

$$
\ln (u) \ln (v) d_{v} \mu(B(v, z)) d_{u} \mu(B(u, y)) d \mu(y) d \mu(z)
$$

и проинтегрировав, получим

$$
\mathrm{E}\left(\ln R_{n, i}^{(k)} \ln R_{n, j}^{(k)} \mid S\right) \leqslant C\left(\max _{k / 2-1 \leqslant k_{1}, k_{2} \leqslant k}\left\{\mathrm{E}\left(-\ln R_{n / 2-k, i}^{\left(k_{1}\right)} \mathrm{E}\left(-\ln R_{n / 2-k, j}^{\left(k_{2}\right)}\right)\right\}\right),\right.
$$

где $C$ - некоторая константа. Выбрав $\varepsilon=\eta / 2$ и подставив неравенства (20) в (25), получим

$$
\mathrm{E}\left(\ln \left(R_{n, i}^{(k)}\right) \ln \left(R_{n, j}^{(k)}\right) \mid S\right) \leqslant \frac{2 C}{\eta} \ln ^{2}\left(\frac{n}{2}-k\right) .
$$

Итак, первое слагаемое в (24) оценивается как

$$
\mathrm{E}\left(\ln \left(R_{n, i}^{(k)}\right) \ln \left(R_{n, j}^{(k)}\right) \mid S\right) \mathrm{P}\{S\}=\mathscr{O}\left(n^{-a \underline{d}}\right) \ln ^{2} n .
$$

Для оценки условного математического ожидания $\mathrm{E}\left(\ln R_{n, i}^{(k)} \ln R_{n, j}^{(k)} \mid \bar{S}\right)$ применим лемму 1 с выбранным значением параметра $a$. Умножив равенства (15) и (16) на

$$
\ln (u) \ln (v) d_{v} \mu(B(v, z)) d_{u} \mu(B(u, y)) d \mu(y) d \mu(z)
$$

и проинтегрировав, получим

$$
\begin{gathered}
\mathrm{E}\left(\ln \left(R_{n, i}^{(k)}\right) \ln \left(R_{n, j}^{(k)}\right) \mid \bar{S}\right)=\left(1+\mathscr{O}\left(n^{-a \underline{d}}\right)\right) \mathrm{E}\left(-\ln R_{n, i}^{(k)}\right) \mathrm{E}\left(-\ln R_{n, j}^{(k)}\right)+\mathscr{O}\left(c^{n^{b}}\right), \\
b>0, \quad 0<c<1 .
\end{gathered}
$$


Подставляя эту оценку и оценку (26) в (24), получим

$$
\operatorname{Cov}\left(-\ln \left(R_{n, i}^{(k)}\right),-\ln \left(R_{n, j}^{(k)}\right)\right)=\mathscr{O}\left(n^{-a \underline{d}}\right) \ln ^{2} n+\mathscr{O}\left(n^{-a \underline{d}}\right) \mathrm{E}\left(-\ln R_{n, i}^{(k)}\right) \mathrm{E}\left(-\ln R_{n, j}^{(k)}\right) .
$$

Применяя неравенства $(20)$ с $\varepsilon=\eta / 2$, получим

$$
\operatorname{Cov}\left(-\ln \left(R_{n, i}^{(k)}\right),-\ln \left(R_{n, j}^{(k)}\right)\right)=\mathscr{O}\left(n^{-a \underline{d}}\right) \ln ^{2} n .
$$

Итак, для любого $\delta<\underline{d} \min \{\underline{d}, 1 / \bar{d}\}$ справедливо (23).

Для завершения доказательства теоремы осталось доказать $(22)$. Для дисперсии $r_{n}^{(k)}$ имеем

$$
\mathrm{D}\left(r_{n}^{(k)}\right)=\frac{1}{n} \mathrm{D}\left(-\ln R_{n, i}^{(k)}\right)+\frac{n-1}{n} \operatorname{Cov}\left(-\ln \left(R_{n, i}^{(k)}\right),-\ln \left(R_{n, j}^{(k)}\right)\right) .
$$

Выберем некоторое $c<\min \left\{\underline{d}^{2}, \underline{d} / \bar{d}, 1\right\}$.

Дисперсию $\mathrm{D}\left(-\ln R_{n, i}^{(k)}\right)$ оценим как

$$
\mathrm{D}\left(-\ln R_{n, i}^{(k)}\right) \leqslant \mathrm{E}\left(\ln ^{2} R_{n, i}^{(k)}\right)
$$

Применяя следствие 4, получим

$$
\mathrm{D}\left(-\ln R_{n, i}^{(k)}\right)=\mathscr{O}\left(\ln ^{2} n\right)
$$

Отсюда и из леммы 3 с параметром $\delta=c$ получаем

$$
\mathrm{D} r_{n}^{(k)}=\mathscr{O}\left(n^{-c}\right) \ln ^{2} n .
$$

СЛЕДСТВИЕ 5. Пусть выполнены условия теоремы 2. Тогда случайные величины $\eta_{n}^{(k)}$, определенные в $(5)$, сходятся по вероятности $\kappa \eta=\eta(\mu, \rho)$ при $n \rightarrow \infty$.

\section{5. Примеры вычисления статэнтропии}

Рассмотрим несколько примеров мер, для которых можно вычислить статэнтропию.

5.1. Гиббсовские меры. Рассмотрим метрическое пространство $S^{\mathbb{N}}$ с метрикой $(8)$, где $S=\{0,1, \ldots, s-1\}$. Пусть $\sigma-$ сдвиг на $S^{\mathbb{N}}$.

Напомним (см., например, [11, теорема 1.2]), что гиббсовская мера $\mu$-это инвариантная мера сдвига $\sigma$, которая строится по заданной гёльдеровской функции $\phi \in C^{\varepsilon}\left(S^{\mathbb{N}}\right)$ таким образом, что для некоторых констант $P, c_{1}>0, c_{2}>0$ выполнено

$$
c_{1} \leqslant \frac{\mu\left(C_{n}(x)\right)}{\exp \left(-P n+\sum_{k=0}^{n-1} \phi\left(\sigma^{k}(x)\right)\right)} \leqslant c_{2} \quad \forall x \in \Omega, \quad n \geqslant 0 .
$$

Пусть $\Omega$ - замькание носителя меры $\mu$. Из определения гиббсовской меры видно, что для $\mu$-почти всех точек $x \in \Omega$ существует

$$
-\lim _{n \rightarrow \infty} \frac{1}{n} \ln \mu\left(C_{n}(x)\right)=P-\int_{\Omega} \phi d \mu .
$$


Применяя вариационный принцип для топологического давления (см., например, [11, теорема 1.22]), получим

$$
P-\int_{\Omega} \phi d \mu=h_{\mu}
$$

где $h_{\mu}$ - энтропия меры $\mu$ (сдвига $\left.\sigma\right)$.

Для метрики (8)

$$
C_{n}(x)=B\left(x, \theta^{-n}\right)=B(x, r), \quad \theta^{-n-1}<r \leqslant \theta^{-n} .
$$

Поэтому для $\mu$-почти всех точек $x \in \Omega$ существует и постоянна точечная размерность $d_{\mu}(x)=h_{\mu} / \ln \theta$. Следовательно, применимо следствие 1 и существует

$$
\eta(\mu, \rho)=\frac{h_{\mu}}{\ln \theta}
$$

Итак, статэнтропия гиббсовской меры совпадает с энтропией с точностью до множителя.

Статистическая оценка (4), (5) является состоятельной при любом $k \geqslant 1$.

Отметим, что в силу вариационного принципа из оценки энтропии легко получить и оценку топологического давления $P$.

5.2. Мера Бернулли. Мера Бернулли является частным случаем гиббсовской меры на метрическом пространстве $\Omega=S^{\mathbb{N}}$, которая задается функцией $\phi(x)=\ln p_{x_{0}}$, где $x=\left(x_{0}, x_{1}, \ldots\right), p_{i}$ - дискретное вероятностное распределение на $S$, давление $P=0$.

Энтропия меры (сдвига) Бернулли хорошо известна:

$$
h_{\mu}=-\sum_{i=0}^{m-1} p_{i} \ln p_{i}
$$

Поэтому

$$
\eta(\mu, \rho)=-\frac{1}{\ln \theta} \sum_{i=0}^{m-1} p_{i} \ln p_{i} .
$$

5.3. Марковская мера. Пусть задана эргодическая марковская цепь с множеством состояний $S=\{0,1, \ldots, s-1\}$, матрицей переходных вероятностей $\left\|a_{i j}\right\|_{0}^{s-1}$ и стационарньг распределением $\left\{p_{i}: i \in S\right\}$ на $S$, где

$$
p_{j}=\sum_{i=0}^{m-1} p_{i} a_{i j}, \quad j \in S .
$$

Обозначим через $\Omega \subset S^{\mathbb{N}}$ множество допустимых последовательностей $\left(a_{x_{i} x_{i+1}}>0\right)$.

Мера $\mu$ определяется как марковская мера по начальному распределению $p_{i}$ и матрице переходных вероятностей $a_{i j}$.

Марковская мера является частным случаем гиббсовской меры на метрическом пространстве $\Omega$, которая также задается функцией $\phi(x)=\ln p_{x_{0}}$, где $x=\left(x_{0}, x_{1}, \ldots\right)$, давление $P=0$. 
Энтропия марковской меры (сдвига) хорошо известна:

$$
h_{\mu}=-\sum_{i=0}^{m-1} \sum_{j=0}^{m-1} p_{i} a_{i j} \ln a_{i j} .
$$

Поэтому

$$
\eta(\mu, \rho)=-\frac{1}{\ln \theta} \sum_{i=0}^{m-1} \sum_{j=0}^{m-1} p_{i} a_{i j} \ln a_{i j}
$$

\section{6. Статэнтропия эргодической динамической системы}

Пусть $X$ - компактное метрическое пространство с метрикой $d, T: X \rightarrow X$ - непрерьвное отображение. Будем считать, что отображение $T$ имеет инвариантную меру $P$, которая является борелевской и вероятностной.

УТВЕРЖДЕНИЕ 3. Пусть для динамической системы $(X, T, P)$ преобразование Т является әргодическим с конечной энтропией $h_{P}(T)$. Тогда

$$
\underline{S H}(T, P)=\overline{S H}(T, P)=\frac{h_{P}(T)}{\ln \theta} .
$$

ДоКАЗАТЕЛЬСТВо. Возьмем конечную случайную величину $\alpha$ и построим символическую динамическую систему $\left(\Omega, \sigma, \mu_{\alpha}\right)$ с метрикой $\rho$, заданной в $(8)$. Поскольку преобразование $T$ является эргодическим, сдвиг $\sigma$ также эргодичен. Поэтому по теореме Шеннона-Макмиллана-Бреймана $[2$, п. 2.10$]$ для $\mu_{\alpha}$-почти всех точек $x \in \Omega$ существует

$$
\lim _{n \rightarrow \infty} \frac{1}{n} \ln \mu_{\alpha}\left(C_{n}(x)\right)=-h(T, \mathscr{A})
$$

где через $h(T, \mathscr{A})$ обозначается энтропия на один знак разбиения $\mathscr{A}$ пространства $X$ на множества $A_{i}=\{x \in X: \alpha(x)=i\}, i \in S$.

Для метрики (8) шары являются цилиндрами, поэтому для $\mu$-почти всех точек $x \in \Omega$ существует и постоянна точечная размерность $d_{\mu}(x)=h(T, \mathscr{A}) / \ln \theta$. Следовательно, применимо следствие 1 и существует статэнтропия

$$
\eta\left(\mu_{\alpha}, \rho\right)=\frac{h(T, \mathscr{A})}{\ln \theta} .
$$

Взяв супремум по всем конечным разбиениям и применив определение энтропии, получим требуемое утверждение.

Итак, для нахождения статэнтропии так же, как и для нахождения метрической энтропии динамической системы, нужно находить случайную величину $\alpha$ (образующее разбиение), для которой реализуется супремум в (10). Существование такой случайной величины устанавливает теорема Колмогорова [3, гл. 14].

Для приближенного вычисления энтропии можно ограничиться выбором достаточно мелкого разбиения. Причем для гомеоморфизмов, разделяющих траектории, образуюшим будет любое разбиение $\mathscr{A} \mathrm{c} \operatorname{diam} A_{i} \leqslant \varepsilon$, где $\varepsilon$ - разделяющая константа [11, 
предложение 2.5]. Напомним, что гомеоморфизм $T: X \rightarrow X$ компактного метрического пространства $(X, d)$ назьвается разделяющим траектории, если существует разделяющая константа $\varepsilon>0$ такая, что если $d\left(T^{n}(x), T^{n}(y)\right)<\varepsilon$ для любого $n \in \mathbb{N}$, то $x=y$.

Таким образом, для нахождения приближенного значения энтропии динамической системы нужно вьполнить следующие шаги:

1) выбрать достаточно мелкое разбиение (чтобы оно было образующим),

2) построить символическую динамическую систему с метрикой эквивалентной (8),

3) выбрать $n$ независимых начальных точек и построить для каждой из них траекторию,

4) применить статистическую оценку (4), (5) к построенным траекториям.

Для эргодических точно размерностных инвариантных мер эта оценка будет состоятельной оценкой энтропии динамической системы (следствие 1).

Приведем несколько примеров динамических систем, у которых меры являются точно размерностными.

ПРИмеР 1. Инвариантная эргодическая мера $\mu$ на репеллере гладкого расширяющего отображения [4, теорема 21.2].

ПРИмеР 2. Инвариантная эргодическая мера $\mu$ на базисном множестве диффеоморфизма, удовлетворяющего аксиоме А [4, теорема 24.2].

ПРимеР 3. Инвариантная эргодическая гиперболическая мера $\mu$ гладкого диффеоморфизма гладкого риманова многообразия [12].

\section{СПИСОК ЦИТИРОВАННОЙ ЛИТЕРАТУРЫ}

[1] Биллингслей П. Эргодическая теория и информация. М.: Мир, 1969.

[2] Мартин Н., Ингленд Дж. Математическая теория энтропии. М.: Мир, 1988.

[3] Синай Я. Г. Введение в эргодическую теорию. Ереван: Изд-во ЕГУ, 1973.

[4] Pesin Ya. Dimension Theory in Dynamical Systems: Contemporary Views and Applications. Chicago: Chicago Univ. Press, 1997.

[5] Добрушин Р. Л.Упрощенный метод экспериментальой оценки энтропии стационарной последовательности // Теория вероятности и ее примен. 1958. Т. 3. № 4. С. 462-464.

[6] Ватутин В.А., Михайлов В. Г. Статистическое оценивание энтропии дискретных случайных величин с большим числом исходов // УМН. 1995. Т. 50. № 5. С. 121-134.

[7] Майоров В.В., Тимофеев Е.А. Состоятельная оценка размерности многообразий и самоподобных фракталов // ЖВМиМФ. 1999. Т. 39. №10. С. 1721-1729.

[8] Майоров В.В., Тимофеев Е. А. Статистическая оценка обобщенных размерностей // Матем. заметки. 2002. Т. 71. № 5. С. 697-712.

[9] Федерер Г. Геометрическая теория меры. М.: Наука, 1987.

[10] Градштейн И. С., Рыжик И. М. Таблицы интегралов, сумм, рядов и произведений. М.: Наука, 1971.

[11] Боуэн Р. Методы символической динамики. М.: Мир, 1979.

[12] Barreira L., Pesin Ya., Schmeling J. Dimension and product structure of hyperbolic measures // IST Preprint 26/99, 1999. 